4 Chideya S, Winston CA, Peloquin CA, et al. Isoniazid, rifampin, ethambutol, and pyrazinamide pharmacokinetics and treatment outcomes among a predominantly HIV-infected cohort of adults with tuberculosis from Botswana. Clin Infect Dis 2009; 48: 1685-1694.

5 Pasipanodya JG, McIlleron H, Burger A, et al. Serum drug concentrations predictive of pulmonary tuberculosis outcomes. J Infect Dis 2013; 208: 1464-1473.

6 Azuma J, Ohno M, Kubota R, et al. NAT2 genotype guided regimen reduces isoniazid-induced liver injury and early treatment failure in the 6-month four-drug standard treatment of tuberculosis: a randomized controlled trial for pharmacogenetics-based therapy. Eur J Clin Pharmacol 2013; 69: 1091-1101.

7 Pasipanodya JG, Srivastava S, Gumbo T. Meta-analysis of clinical studies supports the pharmacokinetic variability hypothesis for acquired drug resistance and failure of antituberculosis therapy. Clin Infect Dis 2012; 55: 169-177.

8 Alsultan A, Peloquin CA. Therapeutic drug monitoring in the treatment of tuberculosis: an update. Drugs 2014; 74: 839-854.

9 Srivastava S, Peloquin CA, Sotgiu G, et al. Therapeutic drug management: is it the future of multidrug-resistant tuberculosis treatment? Eur Respir J 2013; 42: 1449-1453.

10 Lange C, Abubakar I, Alffenaar JW, et al. Management of patients with multidrug-resistant/extensively drug-resistant tuberculosis in Europe: a TBNET consensus statement. Eur Respir J 2014; 44: 23-63.

11 Clinical Laboratory and Standards Institute. Using Proficiency Testing to Improve the Clinical Laboratory; Approved Guideline. 2nd Edn. Wayne, Clinical Laboratory and Standards Institute, 2007.

12 Aarnoutse RE, Verweij-van Wissen CP, van Ewijk-Beneken Kolmer EW, et al. International interlaboratory quality control program for measurement of antiretroviral drugs in plasma. Antimicrob Agents Chemother 2002; 46: $884-886$.

13 Brüggemann RJ, Touw DJ, Aarnoutse RE, et al. International interlaboratory proficiency testing program for measurement of azole antifungal plasma concentrations. Antimicrob Agents Chemother 2009; 53: 303-305.

14 Pranger AD, van Altena R, Aarnoutse RE, et al. Evaluation of moxifloxacin for the treatment of tuberculosis: 3 years of experience. Eur Respir J 2011; 38: 888-894.

15 Sotgiu G, Centis R, D'Ambrosio L, et al. Efficacy, safety and tolerability of linezolid containing regimens in treating MDR-TB and XDR-TB: systematic review and meta-analysis. Eur Respir J 2012; 40: 1430-1442.

\title{
Linezolid for multidrug-resistant tuberculosis in HIV-infected and -uninfected patients
}

To the Editor:

Two recent systematic reviews found that treatment outcomes with regimens containing linezolid for complicated cases of multidrug-resistant (MDR) tuberculosis (TB) are equal to or better than those reported for uncomplicated MDR-TB $[1,2]$ and better than those reported among patients treated for extensively drug-resistant (XDR)-TB [3-6]. Existing data on treatment outcomes with linezolid for MDR-TB are predominantly among HIV-uninfected patients; $<10 \%$ of patients were HIV co-infected in the reviews $[1,2]$. There is concern over the safety of using linezolid within MDR-TB regimens for $\mathrm{HIV}$-infected patients due to underlying HIV-related neuropathy and bone marrow dysfunction as well as a potentially higher incidence of adverse events in patients on antiretroviral therapy (ART), notably anaemia with zidovudine and peripheral neuropathy with stavudine [7]. Given the high rate of HIV/TB co-infection in many settings, we report here our clinical experience with the use of linezolid for treatment of XDR-TB and complicated MDR-TB among HIV-infected and -uninfected patients in community-based programmes supported by Médecins Sans Frontières (MSF) in two high-burden settings in Khayelitsha, South Africa [8], and Mumbai, India [7]. We carried out a retrospective analysis of routinely collected data. Programmes at both sites were approved by either MSF or local university ethics review boards. Due to restricted access to linezolid in South Africa as well as limited capacity for operational research in both programmes, the numbers are small, and data were collected and analysed over a limited time period only.

At time of analysis in August 2013, 34 patients (17 from each site); one with MDR-TB, 16 with pre-XDR-TB (defined as MDR plus resistance to either a fluoroquinolone or an injectable agent) and 17 with XDR-TB) had received individualised regimens with a median of eight drugs (range 7-10), including linezolid, for $\geqslant 1$ month. 18 (53\%) patients started treatment on initial detection of second-line drug resistance; the remainder only after second-line TB treatment appeared to be failing due to continued culture positivity or reconversion from negative to positive. The median second-line treatment duration 
prior to linezolid start in the latter group was 12 months (range 6-49 months). 18 (53\%) patients in the cohort were female; median age was 29 years (range $12-46$ years) and median weight $51 \mathrm{~kg}$ (range 26$94 \mathrm{~kg}$ ). While a starting oral dose of $600 \mathrm{mg}$ linezolid once daily was preferred, two patients with low body mass index $\left(\leqslant 14 \mathrm{~kg} \cdot \mathrm{m}^{-2}\right)$ were started on a lower dose of $300 \mathrm{mg}$ once daily. 19 (56\%) patients were HIV infected (median CD4 count 290 cells $\mathrm{mm}^{-3}$, range $67-490$ cells $\mathrm{mm}^{-3}$ ) and all were on ART (two with stavudine and six with zidovudine-containing regimens) at the time of linezolid start.

The median duration of linezolid treatment among all 34 patients was 8 months (range 1-24 months) at the time of censoring, and at this point, $29(85 \%)$ patients were still alive and on treatment. No patients were lost from treatment (default) but two (6\%) died during treatment; both had been started on linezolid due to failure of second-line therapy. One (HIV uninfected) died within 2 months of starting linezolid and without culture conversion, while the other (HIV infected) died of an unknown cause after 17 months of treatment with linezolid, 14 months after culture conversion. Treatment failed and was withdrawn for a further three (9\%) patients, after a median of 8 months (range 6-10 months), all of whom subsequently died due to clinical progression of TB disease (only one was HIV infected).

Only 28 patients had positive Mycobacterium tuberculosis cultures at linezolid start and, among these, culture conversion was $50 \%$ by 3 months of treatment (fig. 1a). There was no difference in culture conversion over time related to HIV infection (hazard ratio (HR) 1.12, 95\% CI 0.39-3.22; $\mathrm{p}=0.83$ ), resistance pattern (MDR/ pre-XDR versus XDR) (HR 1.27, 95\% CI $0.40-4.00 ; \mathrm{p}=0.69)$ or weight $(<50$ versus $>50 \mathrm{~kg})(\mathrm{HR} 1.64,95 \%$ CI 1.10-4.76; $\mathrm{p}=0.36$ ). Those who started linezolid on initial detection of second-line drug resistance had more rapid culture conversion than those treated after failure of previous treatment (HR 3.33, 95\% CI 1.10-10.20; $\mathrm{p}=0.03$ ), suggesting that individualised regimens containing linezolid should be offered early to improve chance of treatment success rather than reserved as a salvage option later in treatment.

In our cohort of 34 patients, there were 17 episodes of any adverse event attributed to linezolid, reported among $14(41 \%)$ patients: six patients with peripheral neuropathy only, five with anaemia only, two with anaemia and peripheral neuropathy, and one with anaemia and optic neuritis. This incidence was not as high as that previously reported from a cohort of 18 MDR-TB patients treated with linezolid utilising individualised regimens in India [9]. Adverse events were reported pragmatically and it is likely that mild events were not always reported, particularly peripheral neuropathy, which is common among HIV-infected people in these settings [10]. As data were collected retrospectively, this may be an underestimation of the true incidence in our cohort. Encouragingly, six patients showed improvement in adverse event symptoms following dose reduction from $600 \mathrm{mg}$ once daily to $300 \mathrm{mg}$ once daily, whereas seven patients were able to continue linezolid at the same dose after the adverse event was reported. Linezolid was withdrawn in one other patient with severe peripheral neuropathy, who also had prolonged stavudine exposure. Optic neuropathy is reported to be relatively rare but can be irreversible [11]; this event occurred in only one patient after 6 months on linezolid. The patient insisted on continuing linezolid despite persistent visual
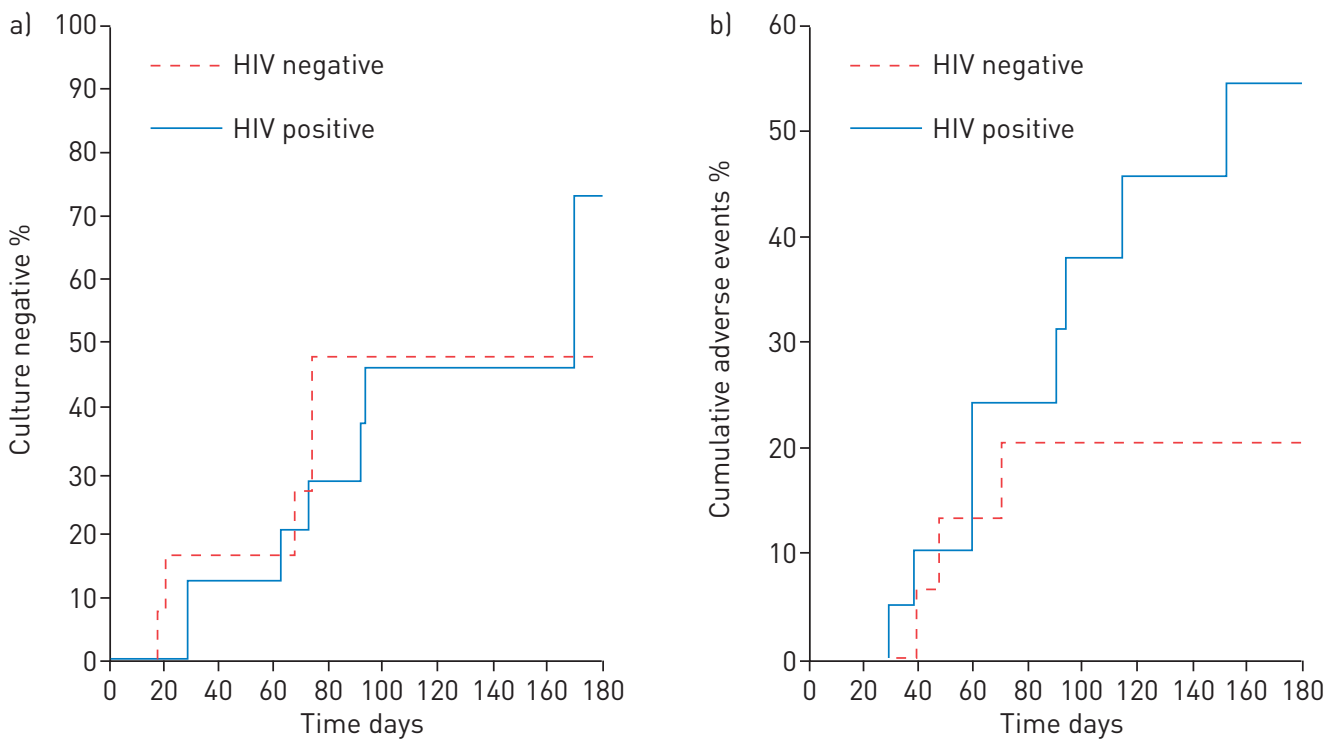

FIGURE 1 Time to event curves. a) Time to culture conversion among patients who were culture positive at linezolid start, by HIV status $(n=28, p=0.83)$. b) Time to any adverse event (anaemia, peripheral neuropathy or optic neuropathy) over 8 months of linezolid treatment, by HIV status $(n=34, p=0.12)$. 
disturbance and extensive counselling and advice to stop, and eventually died of an unknown cause at 17 months. Neither of the two patients who started linezolid at $300 \mathrm{mg}$ once daily experienced any adverse events. It should be noted that while anaemia and neuropathies were occasionally reported to be severe, their occurrence should be viewed in the context of the serious, debilitating and irreversible adverse events associated with other second-line TB medications, such as renal toxicity, deafness and psychosis [12, 13]. Therapeutic drug monitoring has a potential role in the management of complicated MDR-TB cases with limited treatment options. Measurement of serum concentrations of second-line TB drugs may allow individual dosages to be modified in patients at risk of multiple drug-drug interactions, and may minimise adverse events while maintaining optimal exposure to effective treatment $[14,15]$.

We found that while the cumulative incidence of adverse events was high in the whole cohort, HIV-infected patients appeared to have a higher risk of any adverse event associated with linezolid, particularly beyond 3 months of exposure, although this did not reach significance (HR 2.92, 95\% CI 0.9-9.4; $p=0.073$ ) (fig. 1b). This pattern was similar between HIV-infected and uninfected patients for peripheral neuropathy (HR 2.99, 95\% CI 0.60-14.87; $\mathrm{p}=0.18$ ) and anaemia (HR 3.39, 95\% CI 0.68-16.93; $\mathrm{p}=0.14$ ). There was no increased risk of peripheral neuropathy among patients weighing $<50 \mathrm{~kg}$ (HR 1.09, 95\% CI 1.27-4.39; p=0.90) but there appeared to be a higher risk of anaemia with weight $<50 \mathrm{~kg}$ (HR 3.56, 95\% CI 0.81-15.64; p=0.092), particularly among those who were HIV-infected (HR 5.43, 95\% CI 1.25-23.55; p=0.024).

In summary, our data indicate that the rate of culture conversion among pre-XDR and XDR-TB patients treated with linezolid is better than previously reported among XDR cohorts [3-6] and that HIV-infected patients on ART were able to tolerate prolonged linezolid exposure, with dose reduction in some cases. HIV infection was not associated with poorer treatment response, despite low CD4 counts; however, all HIV-infected patients in this cohort were on either first- or second-line ART regimens and most had suppressed viral loads, potentially contributing to encouraging interim outcomes. Routine monitoring for specific adverse events is recommended for all patients receiving linezolid and, although not uncommon, these events may be managed appropriately among both HIV-infected and -uninfected patients, even on an ambulatory basis. patients on ART http://ow.ly/JoPzO

Jennifer Hughes ${ }^{1}$, Petros Isaakidis ${ }^{2}$, Aristomo Andries ${ }^{2}$, Homa Mansoor ${ }^{2}$, Vivian Cox ${ }^{1}$, Graeme Meintjes ${ }^{3,4}$ and Helen $\operatorname{Cox}^{5}$

${ }^{1}$ Médecins Sans Frontières (MSF)/Doctors without Borders, Cape Town, South Africa. ${ }^{2}$ MSF/Doctors without Borders, Mumbai, India. ${ }^{3}$ Clinical Infectious Diseases Research Initiative, Institute of Infectious Disease and Molecular Medicine, and Dept of Medicine, University of Cape Town (UCT), Cape Town, South Africa. ${ }^{4}$ Dept of Medicine, Imperial College London, London, UK. ${ }^{5}$ Division of Medical Microbiology, and Institute for Infectious Disease and Molecular Medicine, UCT, Cape Town, South Africa.

Correspondence: Jennifer Hughes, MSF, PO Box 27401, Rhine Rd, Sea Point, Cape Town, South Africa.

E-mail: msfocb-khayelitsha-tbdoc@brussels.msf.org

Received: July 212014 | Accepted after revision: Feb 032015 | First published online: April 022015

Conflict of interest: None declared.

\section{References}

1 Cox H, Ford N. Linezolid for the treatment of complicated drug-resistant tuberculosis: a systematic review and meta-analysis. Int J Tuberc Lung Dis 2012; 16: 447-454.

2 Sotgiu G, Centis R, D'Ambrosio L, et al. Efficacy, safety and tolerability of linezolid containing regimens in treating MDR-TB and XDR-TB: systematic review and meta-analysis. Eur Respir J 2012; 40: 1430-1442.

3 Dheda K, Shean K, Zumla A, et al. Early treatment outcomes and HIV status of patients with extensively drug-resistant tuberculosis in South Africa: a retrospective cohort study. Lancet 2010; 375: 1798-1807.

4 Jacobson KR, Tierney DB, Jeon CY, et al. Treatment outcomes among patients with extensively drug-resistant tuberculosis: systematic review and meta-analysis. Clin Infect Dis 2010; 51: 6-14.

5 Migliori GB, Sotgiu G, Gandhi NR, et al. Drug resistance beyond extensively drug-resistant tuberculosis: individual patient data meta-analysis. Eur Respir J 2013; 42: 169-179.

6 Falzon D, Gandhi N, Migliori GB, et al. Resistance to fluoroquinolones and second-line injectable drugs: impact on MDR-TB outcomes. Eur Respir J 2013; 42: 156-168.

7 Isaakidis $\mathrm{P}$, Varghese $\mathrm{B}$, Mansoor $\mathrm{H}$, et al. Adverse events among HIV/MDR-TB co-infected patients receiving antiretroviral and second line anti-TB treatment in Mumbai, India. PLoS One 2012; 7: e40781.

8 Cox H, Hughes J, Daniels J, et al. Community-based treatment of drug-resistant tuberculosis in Khayelitsha, South Africa. Int J Tuberc Lung Dis 2014; 18: 441-448.

9 Udwadia ZF, Sen T, Moharil G. Assessment of linezolid efficacy and safety in MDR- and XDR-TB: an Indian perspective. Eur Respir J 2010; 35: 936-938.

10 Robertson K, Kumwenda J, Supparatpinyo K, et al. A multinational study of neurological performance in antiretroviral therapy-naive HIV-1-infected persons in diverse resource-constrained settings. J Neurovirol 2011; 17: 438-447. 
11 Frippiat F, Bergiers C, Michel C, et al. Severe bilateral optic neuritis associated with prolonged linezolid therapy. $J$ Antimicrob Chemother 2004; 53: 1114-1115.

12 Seung KJ, Omatayo DB, Keshavjee S, et al. Early outcomes of MDR-TB treatment in a high HIV-prevalence setting in Southern Africa. PLoS One 2009; 4: e7186.

13 de Jager P, van Altena R. Hearing loss and nephrotoxicity in long-term aminoglycoside treatment in patients with tuberculosis. Int J Tuberc Lung Dis 2002; 6: 622-627.

14 Srivastava S, Peloquin CA, Sotgiu G, et al. Therapeutic drug management: is it the future of multidrug-resistant tuberculosis treatment? Eur Respir J 2013; 42: 1449-1453.

15 Bolhuis MS, van Altena R, van Soolingen D, et al. Clarithromycin increases linezolid exposure in multidrug-resistant tuberculosis patients. Eur Respir J 2013; 42: 1614-1621.

\section{Is Europe ready to reach tuberculosis elimination? An outbreak report from Southern Italy}

To the Editor:

In July 2014, in Rome, the European Respiratory Society and the World Health Organization (WHO) developed a global framework for tuberculosis (TB) elimination in low TB incidence countries, focussed on the concept of TB elimination (fewer than one TB case per million population) [1-5]. Recent studies demonstrated that several "areas" relevant for TB elimination are not fully covered in Europe and in Italy [6, 7].

The aim of the present study was to describe a recent TB outbreak in Puglia (Southern Italy), discuss the practical problems faced by clinicians in managing it correctly, and identify the critical organisational areas impeding proper implementation of WHO and ECDC (European Centre for Disease Prevention and Control) recommendations $[8,9]$.

When deviations from established recommendations $[8,9]$ were observed, a symbol $\left({ }^{d}\right)$ is added in the text.

On December 27, 2013, a 4-year-old child (case A) (fig. 1) was admitted because of long-lasting fever (since November 20) after mononucleosis was excluded. On December 28, chest radiography showed interstitial pneumonia (for which ineffective antibiotic treatment, ceftriaxone plus amikacin, was prescribed) while being tuberculin skin test (TST) negative. Culture was requested (later being positive) and a blood specimen for interferon- $\gamma$ release assay (IGRA) (Quantiferon TB Gold; Cellestis, Carnegie, Australia) was sent to a different laboratory (the only one within the province where the test is available), which resulted positive on January 3, 2014. The computed tomography (CT) confirmed a miliary picture. Standard treatment was initiated with four first-line drugs (isoniazid $(\mathrm{H})$, rifampicin $(\mathrm{R})$, pyrazinamide $(\mathrm{Z})$ and ethambutol (E)). The lack of access to rapid diagnostics $\left({ }^{\mathrm{d} 1}\right)$ in a sputum smear negative case, necessitating a wait for solid culture results $\left({ }^{\mathrm{d} 2}\right)$, contributed to delayed diagnosis, although after admission, adequate treatment was rapidly prescribed. The antibiotic treatment, although including a drug active against Mycobacterium tuberculosis, was considered essential to treat pneumonia.

All adult contacts underwent TST (January 6-10, 2014) and all children attending the same nursery school had IGRA testing (from January 13). Among family members and close contacts of the first circle, 28 adults were tested and three resulted as TST positive and chest radiography negative. Out of 24 health staff tested, all were TST negative. Two children (contacts of case A during his admission) were TST and IGRA negative.

Case A's father recalled that in September 2013, the entire family attended a parish party (involving the members of a local immigrants' association) and a 27 -years-old man had a severe cough. The local health department recorded that in October, a pulmonological medical examination was performed because of bronchitis reported by a man born in Pakistan. He was traced (case B), and underwent chest radiography (multiple lesions on the right lung) and bacteriology (positive for alcohol- and acid-fast bacilli (AFB)). Later, culture resulted positive for M. tuberculosis, while his 24-locus mycobacterial interspersed repetitive units (MIRU) variable number tandem repeats (VNTR) (performed at S. Raffaele Institute, Milan, Italy, in November 2014) resulted different from that of Case A. 